\title{
The Geometry of Musical Rhythm
}

\author{
Godfried Toussaint* \\ School of Computer Science \\ McGill University \\ Montréal, Québec, Canada
}

Dedicated to János Pach on the occasion of his 50th birthday.

\begin{abstract}
Musical rhythm is considered from the point of view of geometry. The interaction between the two fields yields new insights into rhythm and music theory, as well as new problems for research in mathematics and computer science. Recent results are reviewed, and new open problems are proposed.
\end{abstract}

\section{Introduction}

Imagine a clock which has 16 hours marked on its face instead of the usual 12. Assume that the hour and minute hands have been broken off so that only the second-hand remains. Furthermore assume that this clock is running fast so that the second-hand makes a full turn in about 2 seconds. Such a clock is illustrated in Figure 1. Now start the clock ticking at "noon" (16 O'clock) and let it keep running for ever. Finally, strike a bell at positions 16, 3, 6, 10 and 12 , for a total of five strikes per clock cycle. These times are marked with a bell in Figure 1. The resulting pattern rings out a seductive rhythm which, in a short span of fifty years during the last half of the 20th century, has managed to conquer our planet. It is known around the world (mostly) as the Clave Son from Cuba. However, it is common in Africa, and probably travelled from Africa to Cuba with the slaves [65]. In Africa it is traditionally played with an iron bell. In Cuba it is played with two sticks made of hard wood also called claves [43]. More relevant to this paper, there exist purely geometric properties that may explain the world-wide popularity of this clave rhythm [58].

The Clave Son rhythm is usually notated for musicians using standard music notation which affords many ways of expressing a rhythm. Four such examples are given in the top four lines of Figure 2. The fourth line displays the rhythm using the smallest convenient durations of notes and rests. Western music notation is not ideally suited to represent African music [3], [18]. The fifth and sixth lines show two popular ways of representing rhythms that avoid Western notation. The representation on line five is called the Box Notation Method developed by Philip Harland at the University of California in Los Angeles in 1962 and is also

\footnotetext{
* This research was partially supported by NSERC and FCAR. e-mail: godfried@cs.mcgill.ca
} 


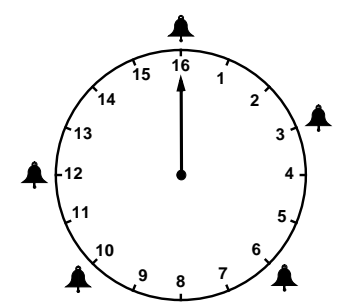

Fig. 1. A clock divided into sixteen equal intervals of time.

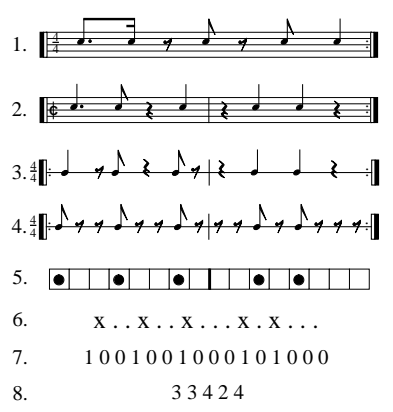

Fig. 2. Eight common ways of representing the clave Son rhythm.

known as TUBS (Time Unit Box System). The TUBS representation is popular among ethnomusicologists [18], and invaluable to percussionists not familiar with Western notation. It is also convenient for experiments in the psychology of rhythm perception, where a common variant of this method is simply to use one symbol for the note and another for the pause [17], as illustrated in line six. In computer science the clave Son might be represented as the 16-bit binary sequence shown on line seven. Finally, line eight depicts the interval length representation of the clave Son, where the numbers denote the lengths (in shortest convenient units) of the durations between consecutive onsets (beginning points in time of notes). The compactness and ease of use in text, of this numerical interval-length representation, are two obvious advantages, but its iconic value is minimal. For a description of additional (more geometric) methods used to represent rhythms see [61].

In this paper several geometric properties of musical rhythm are analysed from the musicological and mathematical points of view. Several connecting bridges between music theory, mathematics, and computer science are illuminated. Furthermore, new open problems at the interface are proposed. 


\section{Measures of Rhythmic Evenness}

Consider the following three $12 / 8$ time rhythms expressed in box-like notation: $[\mathrm{x} \cdot \mathrm{x} \cdot \mathrm{x} \cdot \mathrm{x} \cdot \mathrm{x} \cdot \mathrm{x}],.[\mathrm{x} \cdot \mathrm{x} \cdot \mathrm{x} \mathrm{x} \cdot \mathrm{x} \cdot \mathrm{x} \cdot \mathrm{x}]$ and $[\mathrm{x} \ldots \mathrm{x} \mathrm{x} \ldots \mathrm{x} \mathrm{x}$.$] . It is in-$ tuitively clear that the first rhythm is more even (well spaced) than the second, and the second is more even than the third. In passing we note that the second rhythm is internationally the most well known of all the African timelines. It is traditionally played on an iron bell, and is known on the world scene mainly by its Cuban name Bembé [60]. Traditional rhythms have a tendency to exhibit such properties of evenness to one degree or another. Therefore mathematical measures of evenness, as well as other geometric properties, find application in the new field of mathematical ethnomusicology [9], [62], where they may help to identify, if not explain, cultural preferences of rhythms in traditional music.

\subsection{Maximally even rhythms}

In music theory much attention has been devoted to the study of intervals used in pitch scales [24], but relatively little work has been devoted to the analysis of time duration intervals of rhythm. Two notable exceptions are the books by Simha Arom [3] and Justin London [36]. Clough and Duthett [12] introduced the notion of maximally even sets with respect to scales represented on a circle. According to Block and Douthett [5], Douthet and Entringer went further by constructing several mathematical measures of the amount of evenness contained in a scale (see the discussion on p. 41 of [5]). One of their measures simply adds all the interval arc-lengths (geodesics along the circle) determined by all pairs of pitches in the scale. This definition may be readily transferred to durations in time, of cyclic rhythms represented on a unit circle, as illustrated in Figure 1. However, the measure is too coarse to be useful for comparing rhythm timelines such as those studied in [58] and [60]. Admitedly, the measure does differentiate between rhythms that differ widely from each other. For example, the two four-onset rhythms $[\mathrm{x} \ldots \mathrm{x} \ldots \mathrm{x} \ldots \mathrm{x} \ldots]$ and $[\mathrm{x} . \mathrm{x} . \mathrm{x} \ldots \mathrm{x} \ldots \ldots .$.$] yield evenness$ values of 32 and 23 , respectively, reflecting clearly that the first rhythm is more evenly spaced than the second. However, all six fundamental 4/4 time clave/bell patterns illustrated in Figure 3, and discussed in [58], have an equal evenness value of 48 , and yet the Rumba clave is clearly more uneven than the BossaNova clave. The use of interval chord-lengths (as opposed to geodesic distances), proposed by Block and Douthet [5], yields a more discriminating measure, and is therefore the preferred measure of evenness.

\subsection{Maximizing the sum of distances}

The evenness measure of Block and Douthet [5], which sums all the pairwise straight-line distances of a set of points on the circular lattice, brings up the question of which configurations of points (rhythms) achieve maximum evenness. In fact, this problem had been investigated by the Hungarian mathematician Fejes Tóth [55] some forty years earlier without the restriction of placing the 


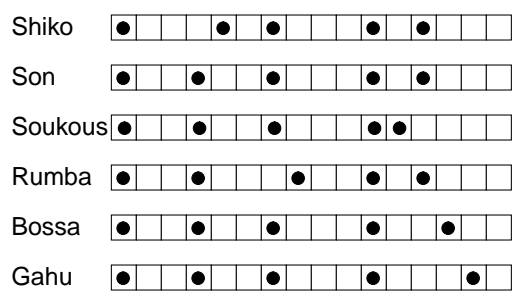

Fig. 3. The six fundamental $4 / 4$ time clave and bell patterns in box notation.

points on the circular lattice. He showed that the sum of the pairwise distances determined by $n$ points in a circle is maximized when the points are the vertices of a regular $n$-gon inscribed on the circle.

The discrete version of this problem, of interest in music theory [5], is a special case of several problems studied in computer science and operations research. In graph theory it is a special case of the maximum-weight clique problem [22]. In operations research it is studied under the umbrella of obnoxious facility location theory. In particular, it is one of the dispersion problems called the discrete $p$ maxian location problem [20], [21]. Because these problems are computationally difficult, researchers have proposed approximation algorithms [30], and heuristics [21], [66], for the general problem, and have sought efficient solutions for simpler special cases of the problem [49], [54].

Fejes Tóth also showed in [55] that in three dimensions four points on the sphere maximize the sum of their pairwise distances when they are the vertices of a regular tetrahedron. The problem remains open for more than four points on the sphere. Some upper and lower bounds on the maximum value that the sum may attain are known. Alexander [1] proved an upper bound of $(2 / 3) n^{2}-(1 / 2)$. It has also been shown that the points must be well spaced in some sense. Stolarsky [53] proved that if $n$ points are placed on the sphere so that the sum of their distances is maximized, then the distance between the closest pair is at least $2 / 3 n$. Additional bounds and references may be found in the survey paper by Chakerian and Klamkin [8].

In 1959 Fejes Tóth [56] asked a more difficult question by relaxing the spherical constraint. He asked for the maximum sum of distances of $n$ points in the plane under the constraint that the diameter of the set is at most one. Pillichshammer [46] found upper bounds on this sum but gave exact solutions only for $n=3,4$, and 5 . For $n=3$ the points form the vertices of an equilateral triangle of unit side lengths. For $n=5$ the points form the vertices of a regular pentagon with unit length diagonals. For $n=4$ the solution may be obtained by placing three points on the vertices of a Reuleaux unit-diameter triangle, and the fourth point at a midpoint of one of the Reuleaux triangle arcs. The problem remains open for more than five points in the plane. In the mathematics literature such problems have also been investigated with the Euclidean distance replaced by the squared Euclidean distance [45], [47], [67]. 

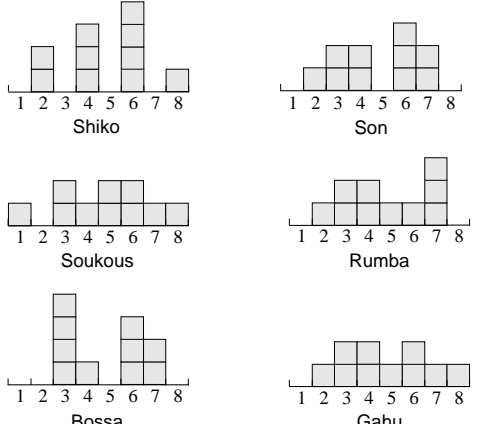

Gahu

Fig. 4. The full-interval histograms of the $4 / 4$ time clave-bell patterns.

\section{Interval Spectra of Rhythms}

Rather than focusing on the sum of all the inter-onset duration intervals of a rhythm, as in the preceeding section, here we examine the shape of the spectrum of the frequencies with which all the inter-onset durations are present in a rhythm. Again we assume rhythms are represented as points on a circle as in Figure 1. In music theory this spectrum is called the interval vector (or fullinterval vector) [39]. For example, the interval vector for the clave Son pattern of Figure 1 is given by $[0,1,2,2,0,3,2,0]$. It is an 8 -dimensional vector because there are eight different possible duration intervals (geodesics on the circle) between pairs of onsets defined on a 16-unit circular lattice. For the clave Son there are 5 onsets (10 pairs of onsets), and therefore the sum of all the vector elements is equal to ten. A more compelling and useful visualization of an interval vector is as a histogram. Figure 4 shows the histograms (interval vectors) of the full-interval sets of all six (4/4)-time clave-bell patterns pictured in Figure 3.

Examination of the six histograms leads to questions of interest in a variety of fields of enquiry: musicology, geometry, combinatorics, and number theory. For example, David Locke [35] has given musicological explanations for the characterization of the Gahu bell pattern (shown at the bottom of Figure 3) as "rhythmically potent", exhibiting a "tricky" quality, creating a "spiralling effect", causing "ambiguity of phrasing" leading to "aural illusions." Comparing the full-interval histogram of the Gahu pattern with the five other histograms in Figure 4 leads to the observation that the Gahu is the only pattern that has a histogram with a maximum height of 2 , and consisting of a single connected component of occupied histogram cells. The only other rhythm with a single connected component is the Rumba, but it has 3 intervals of length 7 . The only other rhythm with maximum height 2 is the Soukous, but it has two connected components because there is no interval of length 2. Only Soukous and Gahu use seven out of the eight possible interval durations. 
The preceeding observations suggest that perhaps other rhythms with relatively uniform (flat) histograms, and few, if any, gaps may be interesting from the musicological point of view as well. Does the histogram shape of the Gahu rhythm play a significant role in the rhythm's special musicological properties? If so, this geometric property could provide a heuristic for the discovery and automatic generation of other "good" rhythms. Such a tool could be used for music composition by computer. With this in mind one may wonder if rhythms exist with the most extreme values possible for these properties. Let us denote the family of all rhythms consisting of $k$ onsets in a time span cycle of $n$ units by $R[k, n]$. In other words $R[k, n]$ consists of all $n$-bit cyclic binary sequences with $k 1$ 's. Thus all the $4 / 4$ time clave-bell patterns in Figure 3 belong to $R[5,16]$.

The first natural question that arizes is whether there exist any rhythms whose inter-onset intervals have perfectly flat histograms of height one with no gaps. This is clearly not possible with $R[5,16]$. Since there are only 8 possible different interval lengths and 10 distance pairs, there must exist at least one histogram cell with height greater than one. The second natural question is whether there exists an $R[5,16]$ rhythm that uses all eight intervals. The answer is yes; one such pattern is $[\mathrm{x} \mathrm{x} \ldots \mathrm{x} . \mathrm{x} \ldots . \mathrm{x} .$.$] with interval vector given$ by $[1,1,1,2,1,2,1,1]$. However, the rhythm $[\mathrm{x} \mathrm{x} \ldots \mathrm{x}$. x . . . . ] belonging to the family $R[4,12]$ depicted on the circle in Figure 5 (a) does have a perfectly flat histogram: every one of the inter-onset intervals occurs exactly once; its interval vector is $[1,1,1,1,1,1]$.

For a rhythm to have "drive" it should not contain silent intervals that are too long, such as the silent interval of length six in Figure 5 (a). One may wonder if there are other rhythms in $R[4,12]$ with interval vectors equal to $[1,1,1,1,1,1]$, and if they exist, are there any with shorter silent gaps. It turns out that the answer is yes. The rhythm $[\mathrm{x} \mathrm{x} . \mathrm{x} \ldots \mathrm{x} \ldots . .$.$] shown in Figure 5$ (b) satisfies all these properties; its longest silent gap is five units.

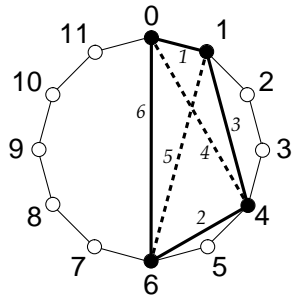

(a)

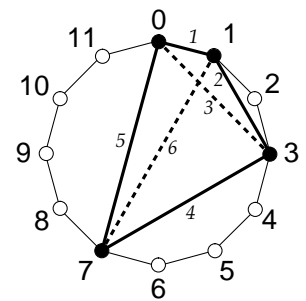

(b)

Fig. 5. Two flat-histogram rhythms. 
A cyclic sequence such as $[\mathrm{x} \times \ldots \mathrm{x} . \mathrm{x} \ldots .$.$] is an instance of a necklace$ with "beads" of two colors [33]; it is also an instance of a bracelet. Two necklaces are considered the same if one can be rotated so that the colors of its beads correspond, one-to-one, with the colors of the other. Two bracelets are considered the same if one can be rotated or turned over (mirror image) so that the colors of their beads are brought into one-to-one correspondence. The rhythms in Figure 5 clearly maintain the same interval vector (histogram) if they are rotated, although this rotation may yield rhythms that sound quite different. Therefore it is useful to distinguish between rhythm-necklaces, and just plain rhythms (necklace instances in a fixed rotational position with respect to the underlying beat). The number of onsets in a rhythm is called the density in combinatorics, and efficient algorithms exist for generating all the necklaces with a specified fixed density [51].

\subsection{Rhythms with specified duration multiplicities}

In 1989 Paul Erdős [19] asked whether one could find $n$ points in the plane (no three on a line and no four on a circle) so that for every $i, i=1, \ldots n-1$ there is a distance determined by these points that occurs exactly $i$ times. Solutions have been found for $2 \leq n \leq 8$. Palásti [44] considered a variant of this problem with further restrictions: no three form a regular triangle, and no one is equidistant from three others. A musical scale whose pitch intervals are determined by points drawn on a circle, and that has the property asked for by Erdös is known in music theory as a deep scale [31]. We will transfer this terminoly from the pitch domain to the time domain and refer to cyclic rhythms with this property as deep rhythms. Deep scales have been studied as early as 1967 by Carlton Gamer [26], [27], and it turns out that the ubiquitous Western diatonic scale is a deep scale. Also, the Bembe rhythm mentioned in the preceeding is a deep rhythm since it is isomorphic to the diatonic scale.

The question posed by Erdős is closely related to the general problem of reconstructing sets from interpoint distances: given a distance multiset, construct all point sets that realize the distance multiset. This problem has a long history in crystallography [34], and more recently in DNA sequencing [52]. Two noncongruent sets of points, such as the two different necklaces of Figures 5, are called homometric if the multisets of their pairwise distances are the same [50]. For an extensive survey and bibliography of this problem see [34]. The special cases relevant to the theory of rhythm, when points lie on a line or circle, have received some attention, and are called the turnpike problem and the beltway problem, respectively [34].

Some existing results on homometric sets on the circular lattice are most relevant to the theory of rhythm. For example many drumming patterns have two sounds (such as the high and low congas) that together tile the lattice. It is known that every $n$-point subset of the regular $2 n$-gon is homometric to its complement [34]. This leads immediately to a simple method for the generation of two-tone tiling rhythms in which each of the two parts is homometric. One 
example is illustrated in Figure 6. It is also known that two rhythms are homometric if, and only if, their complements are [10]. This concept provides another tool that may find use in music composition by computer.

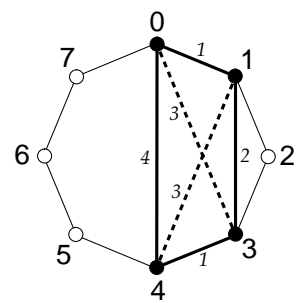

Low Conga

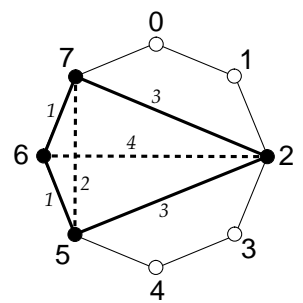

High Conga

Fig. 6. Two complementary homometric rhythms.

\section{Measuring the Similarity of Rhythms}

At the heart of any algorithm for comparing, recognizing or classifying rhythms, lies a measure of the similarity between a pair of rhythms. The type of similarity measure chosen is in part predetermined by the manner in which the rhythm is represented. Furthermore, the design of a measure of similarity is guided by at least two fundamental ideas: what should be measured, and how should it be measured.

There exists a wide variety of methods for measuring the similarity of two rhythms represented by strings of symbols [59]. Indeed the resulting approximate pattern matching problem is a classical problem in pattern recognition and computer science in general [16]. Traditionally similarity between two patterns is measured by a simple template matching operation. More recently similarity has been measured with more powerful and complex functions such as the earth mover's distance [7], [64], weighted geometric matching functions [37], the swap distance [61], and the directed-swap distance [15], [13].

\subsection{Swap distance}

A well known distance measure between two $n$-bit binary sequences is the Hamming distance trivially computed in $O(n)$ time. However, this distance measure is not appropriate for rhythm similarity, when used with a binary-string representation, because although it measures the existence of an onset missmatch, it does not measure how far the missmatch occurs. Furthermore, if a note onset 
of a rhythm is displaced a large distance, the resulting modified rhythm will in general sound more different than if the onset is moved a small distance.

To combat this inherent weakness of the Hamming distance, variants and generalizations of the Hamming distance have been proposed over the years. One early generalization is the edit distance which allows for insertions and deletions of notes. Discussions of the application of the edit-distance to the measurement of similarity in music can be found in Mongeau and Sankoff [40] and Orpen and Huron [42]. A noteworthy more recent generalization is the fuzzy Hamming distance [6] which allows shifting of notes as well as insertions and deletions. Using dynamic programming these distances may be computed in $O\left(n^{2}\right)$ time.

The problem of comparing two binary strings of the same length with the same number of one's suggests an extremely simple edit operation called a swap. A swap is an interchange of a one and a zero that are adjacent to each other in the binary string. Interchanging the position of elements in strings of numbers is a fundamental operation in many sorting algorithms [14]. However, in the sorting literature a swap may interchange non-adjacent elements. When the elements are required to be adjacent, the swap is called a mini-swap or primitive-swap [4]. Here we use the shorter term swap to mean the interchange of two adjacent elements. The swap distance between two rhythms is the minimum number of swaps required to convert one rhythm to the other. The swap distance may be viewed as a simplified version of the generalized Hamming distance [6], where only the shift operation is used, and the cost of the shift is equal to its length. Such a measure of dissimilarity appears to be more appropriate than the Hamming distance between the binary vectors in the context of rhythm similarity [58], [60]. The swap distance may also be viewed as a special case of the more general earth mover's distance (also called transportation distance) used by Typke et al. [64] to measure melodic similarity. Given two sets of points called supply points and demand points, each assigned a weight of material, the earth movers distance measures the minimum amount of work (weight times distance) required to transport material from the supply points to the demand points. No supply point can supply more weight than it has and no demand point receives more weight than it needs. Typke et al. [64] solve this problem using linear programming, a relatively costly computational method. The swap distance is a one dimensional version of the earth mover's distance with all weights equal to one. Furthermore, in the case where both binary sequences have the same number of "one's" (or onsets), there is a one-to-one correspondence between the indices of the ordered onsets of the sequences [32].

The swap distance may of course be computed by actually performing the swaps, but this is inefficient. If $X$ has one's in the first $n / 2$ positions and zero's elsewhere, and if $Y$ has one's in the last $n / 2$ positions and zero's elsewhere, then a quadratic number of swaps would be required. On the other hand, if we compare the distances instead, a much more efficient algorithm results. First scan the binary sequence and store a vector of the $x$-coordinates at which the $k$ onsets occur. Then the swap distance between the two onset-coordinate vectors $U$ and $V$ with $k$ onsets is given by: 


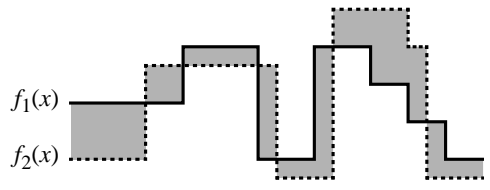

Fig. 7. Two melodies as rectilinear pitch-duration functions of time.

$$
d_{S W A P}(U, V)=\sum_{i=1}^{k}\left|u_{i}-v_{i}\right|
$$

Computing $U$ and $V$ from $X$ and $Y$ is done trivially in $O(n)$ time with a simple scan. Therefore $O(n)$ time suffices to compute $d_{S W A P}(U, V)$, resulting in a large gain over the linear or dynamic programming algorithms.

\section{Introducing Melody into Rhythm}

ÓMaidín [41] proposed a geometric measure of the distance between two melodies modelled as monotonic pitch-duration rectilinear functions of time as depicted in Fig. 7. ÓMaidín measures the distance between the two melodies by the area between the two polygonal chains (shown shaded in Fig. 7). Note that if the area under each melody contour is equal to one, the functions can be viewed as probability distributions, and in this case ÓMaidín's measure is identical to the classical Kolmogorov variational distance used to measure the difference between two probability distributions [57]. If the number of vertices (vertical and horizontal segments) of the two polygonal chains is $n$ then it is trivial to compute ÓMaidín's distance in $O(n)$ time using a line-sweep algorithm.

In a more general setting, such as music information retrieval systems, we are given a short query segment of music, denoted by the polygonal chain $Q=$ $\left(q_{1}, q_{2}, \ldots, q_{m}\right)$, and a longer stored segment $S=\left(s_{1}, s_{2}, \ldots, s_{n}\right)$, where $m<n$. Furthermore, the query segment may be presented in a different key (transposed in the vertical direction) and in a different tempo (scaled linearly in the horizontal direction). Note that the number of keys (horizontal levels) is a small finite constant. Time is also quantized into fixed intervals (such as eighth or sixteenth notes). In this context it is desired to compute the minimum area between the two contours under vertical translations and horizontal scaling of the query. Francu and Nevill-Manning [25] claim that this distance measure can be computed in $O(m n)$ time but they do not describe their algorithm in detail. 


\section{New Open Problems}

Let us assume that we are given a circular lattice with $n$ points (evenly spaced), and we would like to create a rhythm consisting of $k$ onsets by choosing $k$ of these $n$ lattice points. For example, perhaps $n=16$ and $k=5$ as in Figure 1. Furthermore we would like to select the $k$ onsets that maximize the sum of the lengths of all pairwise chords between these onsets. Evaluating all $n$-choose- $k$ subsets may in general be too costly. However, interesting rhythms often have additional musicological constraints that may be couched in a geometric setting [3], and may permit simpler solutions. One may also consider an approximation method using the following snap heuristic: construct a regular $k$-gon with one vertex coincident with one lattice point, and then move the remaining onset points to their nearest lattice points. One would expect such a rhythm to have high evenness value. How close to optimal is this procedure?

The two sequences shown in Figure 5 are the only possible rhythm bracelets with flat histograms, for any values of $k$ greater than three [48]. Therefore in order to be able to generate additional rhythms with near-flat histograms the constraints outlined in the preceeding need to be relaxed. We may proceed in several directions. For example, it is desirable for timelines that can be played fast, and that "roll along" (such as the Gahu already discussed), that the rhythm contain silent gaps that are neither too short nor too long. Therefore it would be desirable to be able to efficiently generate rhythms that either contain completely prescribed histogram shapes, or have geometric constraints on their shapes, and to find good approximations when such rhythms do not exist.

The analysis of cyclic rhythms suggests another variant of the question asked by Erdös. First note that if a rhythm $\mathrm{R}[k, n]$ has $k \leq n / 2$, then a solution to Erdős' problem always exists: simply place points at positions $0,1,2, \ldots, k$. However, as mentioned in the preceeding, from the musicological point of view it may be desirable sometimes not to allow empty semicircles. These constraints suggest the following problem. Is it possible to have $k$ points on a circular lattice of $n$ points so that for every $i, i=k_{s}, k_{s+1}, \ldots, k_{f}$ ( $s$ and $f$ are pre-specified) there is a geodesic distance that occurs exactly $i$ times, with (or without) the further restriction that there is no empty semicircle?

The preceeding discussion on the swap distance was restricted to comparing two linear strings. However, many rhythms such as the timelines considered here are cyclic, and there are applications, such as music information retrieval, where it is desired to compute the best alignement of two cyclic rhythms over all possible rotations. In other words, it is of interest to compute the distance between two rhythms minimized over all possible rotations of one with respect to the other. Some work has been done with cyclic string matching for several definitions of string similarity [28], [38], [11]. Consider two binary sequences of length $n$ and density $k$ ( $k$ ones and $(n-k)$ zeros). It is desired to compute the minimum swap distance between the two strings under all possible alignments. I call this distance the cyclic swap-distance or also the necklace swap-distance, since it is the swap distance between two necklaces. From the preceeding discussion it follows that the cyclic swap distance may be computed in $O\left(n^{2}\right)$ time by using 
the linear-time algorithm in each of the $n$ possible alignment positions of the two rhythms. Note that swaps may be performed in whatever direction (clockwise or counter-clockwise) yields the fewest swaps. Can the cyclic swap distance be computed in $o\left(n^{2}\right)$ time? In contrast, if the swap distance is replaced with the Hamming distance, then the cyclic (or necklace) Hamming distance may be computed in $O(n \log n)$ time with the Fast Fourier Transform [23], [29].

The work of ÓMaidín [41] and Francu and Nevill-Manning [25] suggests several interesting open problems. In the acoustic signal domain the vertical transposition is continuous rather than discrete. The same can be said for the time axis. What is the complexity of computing the minimum area between a query $Q=\left(q_{1}, q_{2}, \ldots, q_{m}\right)$ and a longer stored segment $S=\left(s_{1}, s_{2}, \ldots, s_{n}\right)$ under these more general conditions?

A simpler variant of the melody similarity problem concerns acoustic rhythmic melodies, i.e., cyclic rhythms with notes that have pitch as a continuous variable. Here we assume two rhythmic melodies of the same length are to be compared. Since the melodies are cyclic rhythms they can be represented as closed curves on the surface of a cylinder. What is the complexity of computing the minimum area between the two rectilinear polygonal chains under rotations around the cylinder and translations along the length of the cylinder? Aloupis et al. [2] present an $O(n)$ time algorithm to compute this measure if rotations are not allowed, and an $O\left(n^{2} \log n\right)$ time algorithm for unrestricted motions (rotations around the cylinder and translations along the length of the cylinder). Can this complexity be improved?

In the preceeding sections several tools were pointed out that can be used for computer composition. We close the paper by mentioning one additional tool for automatically selecting rhythm timelines that can be used for generating new music. In [63] it is shown that the Euclidean algorithm for finding the greatest common divisor of two numbers can be used to generate very good rhythm timelines when the two numbers that serve as input to the Euclidean algorithm are the number of onsets $(k)$ and the time-span $(n)$, respectively, of the desired rhythm. The resulting rhythms, called Euclidean rhythms, are particularly attractive when $k$ and $n$ are relatively prime. What is the relation between Euclidean rhythms and maximally even rhythms under the different definitions of even considered in the preceeding?

\section{References}

1. R. Alexander. On the sum of distances between $n$ points on a sphere. Acta. Math. Acad. Sci. Hungar., 23:443-448, 1972.

2. Greg Aloupis, Thomas Fevens, Stefan Langerman, Tomomi Matsui, Antonio Mesa, Yurai Nuñez, David Rappaport, and Godfried Toussaint. Computing a geometric measure of the similarity between two melodies. In Proc. 15th Canadian Conf. Computational Geometry, pages 81-84, Dalhousie University, Halifax, Nova Scotia, Canada, August 11-13 2003.

3. Simha Arom. African Polyphony and Polyrhythm. Cambridge University Press, Cambridge, England, 1991. 
4. Therese Biedl, Timothy Chan, Erik D. Demaine, Rudolf Fleischer, Mordecai Golin, James A. King, and Ian Munro. Fun-sort - or the chaos of unordered binary search. Discrete Applied Mathematics, 144(Issue 3):231-236, December 2004.

5. Steven Block and Jack Douthett. Vector products and intervallic weighting. Journal of Music Theory, 38:21-41, 1994.

6. Abraham Bookstein, Vladimir A. Kulyukin, and Timo Raita. Generalized Hamming distance. Information Retrieval, 5(4):353-375, 2002.

7. Sung-Hyuk Cha and Sargur N. Srihari. On measuring the distance between histograms. Pattern Recognition, 35:1355-1370, 2002.

8. G. D. Chakerian and M. S. Klamkin. Inequalities for sums of distances. The American Mathematical Monthly, 80(9):1009-1017, November 1973.

9. M. Chemillier. Ethnomusicology, ethnomathematics. The logic underlying orally transmitted artistic practices. In G. Assayag, H. G. Feichtinger, and J. F. Rodrigues, editors, Mathematics and Music, pages 161-183. Springer, 2002.

10. C. Chieh. Analysis of cyclotomic sets. Zeitschrift Kristallographie, 150:261-277, 1979.

11. Kuo-Liang Chung. An improved algorithm for solving the banded cyclic string-tostring correction problem. Theoretical Computer Science, 201:275-279, 1998.

12. J. Clough and J. Douthett. Maximally even sets. Journal of Music Theory, 35:93173,1991

13. Justin Colannino and Godfried Toussaint. An algorithm for computing the restriction scaffold assignment problem in computational biology. Information Processing Letters, 2005. in press.

14. N. G. de Bruijn. Sorting by means of swapping. Discrete Mathematics, 9:333-339, 1974.

15. Miguel Díaz-Bañez, Giovanna Farigu, Francisco Gómez, David Rappaport, and Godfried T. Toussaint. El compás flamenco: a phylogenetic analysis. In Proc. BRIDGES: Mathematical Connections in Art, Music and Science, Southwestern College, Kansas, July 30 - August 12004.

16. Richard O. Duda, Peter E. Hart, and David G. Stork. Pattern Classification. John Wiley and Sons, Inc., New York, 2001.

17. Douglas Eck. A positive-evidence model for classifying rhythmical patterns. Technical Report IDSIA-09-00, Instituto Dalle Molle di studi sull'intelligenza artificiale, Manno, Switzerland, 2000.

18. Laz E. N. Ekwueme. Concepts in African musical theory. Journal of Black Studies, 5(1):35-64, September 1974.

19. Paul Erdős. Distances with specified multiplicities. American Math. Monthly, 96:447, 1989.

20. E. Erkut, T. Baptie, and B. von Hohenbalken. The discrete $p$-maxian location problem. Computers in Operations Research, 17(1):51-61, 1990.

21. E. Erkut, T. Ulkusal, and O. Yenicerioglu. A comparison of $p$-dispersion heuristics. Computers in Operations Research, 21(10):1103-1113, 1994.

22. Sándor P. Fekete and Henk Meijer. Maximum dispersion and geometric maximum weight cliques. Algorithmica, 38:501-511, 2004.

23. M. J. Fisher and M. S. Patterson. String matching and other products. In Richard M. Karp, editor, Complexity of Computation, volume 7, pages 113-125. SIAM-AMS, 1974.

24. A. Forte. The Structure of Atonal Music. Yale Univ. Press, New Haven, 1973.

25. Cristian Francu and Craig G. Nevill-Manning. Distance metrics and indexing strategies for a digital library of popular music. In Proceedings of the IEEE International Conference on Multimedia and EXPO (II), 2000. 
26. Carlton Gamer. Deep scales and difference sets in equal-tempered systems. In Proceedings of the Second Annual Conference of the American Society of University Composers, pages 113-122, 1967.

27. Carlton Gamer. Some combinational resources of equal-tempered systems. Journal of Music Theory, 11:32-59, 1967.

28. J. Gregor and M. G. Thomason. Efficient dynamic programming alignment of cyclic strings by shift elimination. Pattern Recognition, 29:1179-1185, 1996.

29. D. Gusfield. Algorithms on Strings, Trees, and Sequences: Computer Science and Computational Biology. Cambridge University Press, Cambridge, 1997.

30. R. Hassin, S. Rubinstein, and A. Tamir. Approximation algorithms for maximum dispersion. Operations Research Letters, 21:133-137, 1997.

31. Timothy A. Johnson. Foundations of Diatonic Theory: A Mathematically Based Approach to Music Fundamentals. Key College Publishing, Emeryville, California, 2003.

32. Richard M. Karp and Shou-Yen R. Li. Two special cases of the assignment problem. Discrete Mathematics, 13:129-142, 1975.

33. Michael Keith. From Polychords to Pólya: Adventures in Musical Combinatorics. Vinculum Press, Princeton, 1991.

34. Paul Lemke, Steven S. Skiena, and Warren D. Smith. Reconstructing sets from interpoint distances. Tech. Rept. DIMACS-2002-37, 2002.

35. David Locke. Drum Gahu: An Introduction to African Rhythm. White Cliffs Media, Gilsum, New Hampshire, 1998.

36. Justin London. Hearing in Time. Oxford University Press, Oxford, England, 2004.

37. Anna Lubiw and Luke Tanur. Pattern matching in polyphonic music as a weighted geometric translation problem. In Proceedings of the Fifth International Symposium on Music Information Retrieval, pages 289-296, Barcelona, Spain, October 2004 .

38. Maurice Maes. On a cyclic string-to-string correction problem. Information Processing Letters, 35:73-78, 1990.

39. Brian J. McCartin. Prelude to musical geometry. The College Mathematics Journal, 29(5):354-370, 1998.

40. M. Mongeau and D. Sankoff. Comparison of musical sequences. Computers and the Humanities, 24:161-175, 1990.

41. D. ÓMaidín. A geometrical algorithm for melodic difference. Computing in Musicology, 11:65-72, 1998.

42. Keith S. Orpen and David Huron. Measurement of similarity in music: A quantitative approach for non-parametric representations. In Computers in Music Research, volume 4, pages 1-44. 1992.

43. Fernando Ortiz. La Clave. Editorial Letras Cubanas, La Habana, Cuba, 1995.

44. Ilona Palásti. A distance problem of Paul Erdős with some further restrictions. Discrete Mathematics, 76:155-156, 1989.

45. F. Pillichshammer. On the sum of squared distances in the Euclidean plane. Arch. Math., 74:472-480, 2000.

46. F. Pillichshammer. A note on the sum of distances in the Euclidean plane. Arch. Math., 77:195-199, 2001.

47. F. Pillichshammer. On extremal point distributions in the Euclidean plane. Acta. Math. Acad. Sci. Hungar., 98(4):311-321, 2003.

48. John Rahn. Basic Atonal Theory. Schirmer, 1980.

49. S. S. Ravi, D. J. Rosenkrantz, and G. K. Tayi. Heuristic and special case algorithms for dispersion problems. Operations Research, 42(2):299-310, March-April 1994 
50. Joseph Rosenblatt and Paul Seymour. The structure of homometric sets. SIAM Journal of Algebraic and Discrete Methods, 3:343-350, 1982.

51. Frank Ruskey and Joe Sawada. An efficient algorithm for generating necklaces with fixed density. SIAM Journal of Computing, 29(2):671-684, 1999.

52. S. S. Skiena and G. Sundaram. A partial digest approach to restriction site mapping. Bulletin of Mathematical Biology, 56:275-294, 1994.

53. Keneth B. Stolarsky. Spherical distributions of N points with maximal distance sums are well spaced. Proceedings of the American Mathematical Society, 48(1):203-206, March 1975.

54. Arie Tamir. Comments on the paper: "Heuristic and special case algorithms for dispersion problems" by S. S. Ravi, D. J. Rosenkrantz, and G. K. Tayi. Operations Research, 46:157-158, 1998.

55. L. Fejes Tóth. On the sum of distances determined by a pointset. Acta. Math. Acad. Sci. Hungar., 7:397-401, 1956.

56. L. Fejes Tóth. Über eine Punktverteilung auf der Kugel. Acta. Math. Acad. Sci. Hungar., 10:13-19, 1959.

57. Godfried T. Toussaint. Sharper lower bounds for discrimination information in terms of variation. IEEE Transactions on Information Theory, pages 99-100, January 1975.

58. Godfried T. Toussaint. A mathematical analysis of African, Brazilian, and Cuban clave rhythms. In Proc. of BRIDGES: Mathematical Connections in Art, Music and Science, pages 157-168, Towson University, MD, July 27-29 2002.

59. Godfried T. Toussaint. Algorithmic, geometric, and combinatorial problems in computational music theory. In Proceedings of X Encuentros de Geometria Computacional, pages 101-107, University of Sevilla, Sevilla, Spain, June 16-17 2003.

60. Godfried T. Toussaint. Classification and phylogenetic analysis of African ternary rhythm timelines. In Proceedings of BRIDGES: Mathematical Connections in Art, Music and Science, pages 25-36, Granada, Spain, July 23-27 2003.

61. Godfried T. Toussaint. A comparison of rhythmic similarity measures. In Proc. 5th International Conference on Music Information Retrieval, pages 242-245, Barcelona, Spain, October 10-14 2004. Universitat Pompeu Fabra.

62. Godfried T. Toussaint. A mathematical measure of preference in African rhythm. In Abstracts of Papers Presented to the American Mathematical Society, volume 25, page 248, Phoenix, January 7-10 2004. American Mathematical Society.

63. Godfried T. Toussaint. The Euclidean algorithm generates traditional musical rhythms. In Proc. of BRIDGES: Mathematical Connections in Art, Music and Science, Banff, Canada, July 31 - August 32005.

64. Rainer Typke, Panos Giannopoulos, Remco C. Veltkamp, Frans Wiering, and René van Oostrum. Using transportation distances for measuring melodic similarity. In Holger H. Hoos and David Bainbridge, editors, Proceedings of the Fourth International Symposium on Music Information Retrieval, pages 107-114, Johns Hopkins University, Baltimore, 2003.

65. Chris Washburne. Clave: The African roots of salsa. In Kalinda!: Newsletter for the Center for Black Music Research. Columbia University, New York, 1995. Fall-Issue.

66. Douglas J. White. The maximal dispersion problem and the "first point outside the neighbourhood heuristic. Computers in Operations Research, 18(1):43-50, 1991.

67. H. S. Witsenhausen. On the maximum of the sum of squared distances under a diameter constraint. The American Mathematical Monthly, 81(10):1100-1101, December 1974. 